\title{
Comparison of the BCI Performance between the Semitransparent Face Pattern and the Traditional Face Pattern
}

\author{
Jiao Cheng, Jing Jin, and Xingyu Wang \\ Key Laboratory of Advanced Control and Optimization for Chemical Processes, Ministry of Education, \\ East China University of Science and Technology, Shanghai, China \\ Correspondence should be addressed to Jing Jin; jinjingat@gmail.com
}

Received 18 October 2016; Revised 26 January 2017; Accepted 16 March 2017; Published 9 April 2017

Academic Editor: Mikhail A. Lebedev

Copyright (C) 2017 Jiao Cheng et al. This is an open access article distributed under the Creative Commons Attribution License, which permits unrestricted use, distribution, and reproduction in any medium, provided the original work is properly cited.

Brain-computer interface (BCI) systems allow users to communicate with the external world by recognizing the brain activity without the assistance of the peripheral motor nervous system. P300-based BCI is one of the most common used BCI systems that can obtain high classification accuracy and information transfer rate (ITR). Face stimuli can result in large event-related potentials and improve the performance of P300-based BCI. However, previous studies on face stimuli focused mainly on the effect of various face types (i.e., face expression, face familiarity, and multifaces) on the BCI performance. Studies on the influence of face transparency differences are scarce. Therefore, we investigated the effect of semitransparent face pattern (STF-P) (the subject could see the target character when the stimuli were flashed) and traditional face pattern (F-P) (the subject could not see the target character when the stimuli were flashed) on the BCI performance from the transparency perspective. Results showed that STF-P obtained significantly higher classification accuracy and ITR than those of F-P $(p<0.05)$.

\section{Introduction}

Brain-computer interface (BCI) is a technology that allow users to communicate with others or control external devices via brain activity alone [1-3]. BCI directly measures brain activities usually based on electroencephalography (EEG) recorded noninvasively through electrodes placed on the surface of the head [4]. The intention of users can be recognized by analyzing the EEG signals of various mental tasks $[5,6]$ which can help these users directly control external devices through brain activities.

P300-based BCI is one of the most common used BCI systems presented by Farwell and Donchin [7], and this system uses the flash letter pattern. Over the past two decades, the "flash only" paradigm in which the target reverses color or is briefly masked by a solid box [8] is usually used as stimulus to elicit P300 potentials. However, recent studies are focused mostly on a new stimulus that the target is overlapped with a famous face. Such stimuli actually yield better performance compared with the conventional flash only pattern through numerous experiments [8-12]. This result is due to that the face stimuli can elicit other eventrelated potential (ERP) components not restricted to the P300 components (such as vertex positive potential (VPP), $\mathrm{N} 170, \mathrm{~N} 200$, and N400), and these potentials also contribute to the classification accuracy. Zhang et al. [11, 12] reported that VPP and N170 can help improve classification accuracy with stimuli that change to faces. Jin et al. $[8,9,13]$ also reported that N400 significantly contributes to improving the classification accuracy in ERP-based BCI system. Currently, a variety of face patterns have been proposed by numerous researchers. Jin et al. $[8,10]$ presented various types of face paradigms (neutral face, smiling face, shaking neutral face, and shaking smiling face paradigms) and compared multifaces using various familiar faces with single faces. The results indicated that the performance of the ERPbased BCI is enhanced by these face stimuli. Kaufmann et al. [9] introduced face stimuli transparently superimposed on characters in comparison with the flash pattern. Their result showed that such stimuli can generate higher ERP amplitudes and obtain higher classification accuracy than those of the flash letter pattern. However, previous studies 


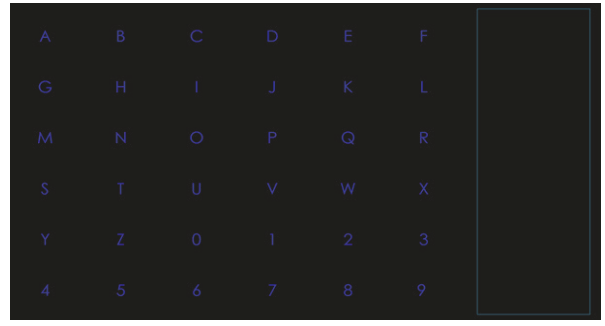

(a)

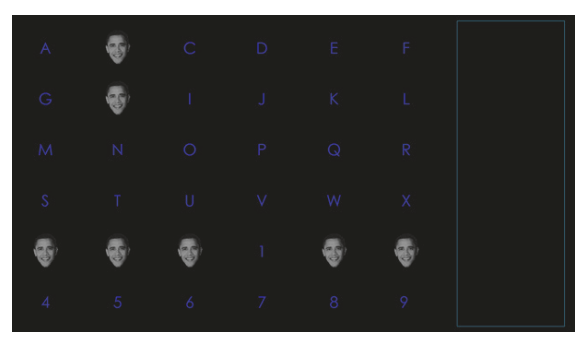

(b)

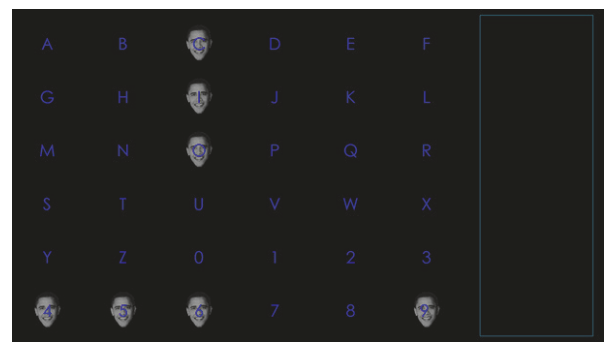

(c)

FIGURE 1: The display presented to subjects. (a) $6 \times 6$ matrix displayed in the monitor; (b) face pattern (F-P); (c) semitransparent face pattern (STF-P).

on face stimuli focused mainly on the effect of various face types (i.e., face expression, face familiarity, and multifaces) on the BCI performance $[8,10,14]$. Studies on the influence of face transparency differences are scarce. Therefore, we investigated the effect of semitransparent face pattern (STF$\mathrm{P}$ ) (the subject could see the target character when the stimuli were flashed) and traditional face pattern (F-P) (the subject could not see the target character when the stimuli were flashed) on the BCI performance. Semitransparent faces can increase the psychological salience of the stimulus and allow for uninterrupted attention. Thus, we hypothesized that the persistent visible target character could help subjects focus on the target, increase N200 and N400 amplitudes, and improve the performance of ERP-based BCI using semitransparent face stimuli.

\section{Methods}

2.1. Subjects. Ten healthy subjects ( 2 females and 8 males, aged 22-26 years with the mean age of 23.5) participated in this study. The native language of the subjects is Mandarin Chinese. In addition, these subjects are all right-handed and have no known neurological disorders. They signed written consent form prior to the experiment, and the local ethics committee approved the consent form and experimental procedure before any subjects participated. Furthermore, all subjects had no previous BCI experiences.

2.2. Stimuli and Procedure. The subjects sat in a chair in front of the monitor, which displayed a $6 \times 6$ matrix with characters and numbers (Figure 1(a)) [10]. They were required to silently count the number of times the target flashed and avoid unnecessary movements. In this study, two paradigms were presented to the subjects. The parameters of the two paradigms (i.e., character size, intercharacter distance, background color, and stimulus brightness) were kept constant, except stimulus transparency. Accordingly, the counterbalance of the paradigm presentation could be obtained. In the first paradigm, the face stimulus concealed the target character, and the subject could not see the target character during the time the stimulus was on (Figure 1(b)). We called this paradigm as the traditional face pattern ( $\mathrm{F}$ $\mathrm{P})$. In the second paradigm, the face stimulus was made semitransparent, and the subject could see the target character during the time the stimuli were on (Figure $1(\mathrm{c})$ ). We called this paradigm as semitransparent F-P (STF-P). The subjects were tasked to count the number of times the target flashed. The flash configuration was based on binomial coefficients $[15,16]$. The binomial coefficients were based on $C(n, k)=n ! /(k !(n-k) !), 0 \leq k \leq n$, where $n$ represents the number of flashes per trial and $k$ represents the number of flashes per trial for each element in the matrix. We chose the combination of $C(12,2)$ to denote the 12flash pattern. Table 1 shows the configuration of the 12-flash pattern combination with 36 flash pattern pairs. The positions in Table 1 corresponded to the positions of the 36 characters in a $6 \times 6$ matrix.

Each subject was required to complete two paradigms (F$\mathrm{P}$ and STF-P) on the same day. Each paradigm contained one offline block and one online block. The order of the paradigms F-P and STF-P was counterbalanced throughout the experiment (five of ten subjects did the F-P first). During the offline period, each paradigm consisted of three offline runs called one offline block, and each offline run contained 5 target characters that would be spelled by the subjects without any rest. The subjects had $3 \mathrm{~min}$ rest between each offline run. In addition, each target character was identified through 16 trials, and each trial was composed of 12 flashes. No feedback 
TABLE 1: Configuration of the 12-flash pattern combination.

\begin{tabular}{lccccc}
\hline 1,4 & 1,5 & 1,6 & 1,7 & 1,8 & 1,9 \\
2,10 & 2,5 & 2,6 & 2,7 & 2,8 & 2,9 \\
3,10 & 3,11 & 3,6 & 3,7 & 3,8 & 3,9 \\
4,10 & 4,11 & 4,12 & 4,7 & 4,8 & 4,9 \\
5,10 & 5,11 & 5,12 & 1,10 & 5,8 & 5,9 \\
6,10 & 6,11 & 6,12 & 3,12 & 2,11 & 6,9 \\
\hline
\end{tabular}

Notes. We named these 12 -flash groups as "flash ${ }_{1}$, flash $_{2}, \ldots$, flash $_{12}$." The numbers in the table represent the target character of the 12 flashes. The same number in the configuration would simultaneously present stimuli. For example, letter "A" was flashed in flash ${ }_{1}$ and flash $\mathrm{H}_{4}$. The italicized numbers represent the positions that simultaneously presented face stimuli during flash $_{11}$.

would be presented to the subjects in the offline experiment. However, during the online period, each paradigm only had one online run called one online block. The number of trials for recognizing each target character was selected automatically by an adaptive strategy $[17,18]$, and each trial was also composed of 12 flashes. The subjects were required to spell 36 target characters without any rest during the online period, and the system would promptly present the online result whenever the classifier recognized the target character. The stimulus on time was $100 \mathrm{~ms}$ and the stimulus onset asynchrony was $250 \mathrm{~ms}$ throughout the offline and online experiments. Moreover, an italicized number was used to prompt the subjects of the next target character before each run started, and they had $4 \mathrm{~s}$ for target selection. In addition, after finishing the offline experiment, subjects had 4 min rest to prepare for the following online experiment. Copy spelling task was used in the offline and online experiments.

2.3. Calibration. We acquired the EEG signals recorded with g.USBamp and g.EEGcap (Guger Technologies, Graz, Austria) with a sensitivity of $100 \mu \mathrm{V}$, band-pass filtered between 0.5 and $30 \mathrm{~Hz}$, and sampled at $256 \mathrm{~Hz}$. A total of 16 corresponding electrode positions were selected in the experiments according to the International 10-20 System (Figure 2) [8]. These positions were Fz, FC1, FC2, C3, Cz, C4, P3, Pz, P4, $\mathrm{O} 1, \mathrm{Oz}, \mathrm{O} 2, \mathrm{P} 7, \mathrm{P} 8, \mathrm{~F} 3$, and $\mathrm{F} 4 . \mathrm{FPz}$ was used as the ground electrode, while right mastoid (A) was used as the reference electrode. These selected electrodes were used to keep track of the EEG signals.

2.4. Feature Extraction Procedure. Feature extraction is an effective method for reducing dimensionality and amount of required computations [18]. In this study, the third-order Butterworth band-pass filter was used to filter the EEG signals; the high pass was $0.5 \mathrm{~Hz}$ and low pass was $30 \mathrm{~Hz}$. In addition, we downsampled the EEG signals from $256 \mathrm{~Hz}$ to $36.6 \mathrm{~Hz}$ by selecting every seventh sample from the filtered EEG. Consequently, we obtained the feature vector with the size of $16 \times 29$ (16 represents the number of the channels and 29 denotes the time points). In addition, winsorizing was used to remove the electrooculogram interference signals. The 10th and 90th percentiles were computed for each sample. Amplitude values lying below the 10th percentile or above the

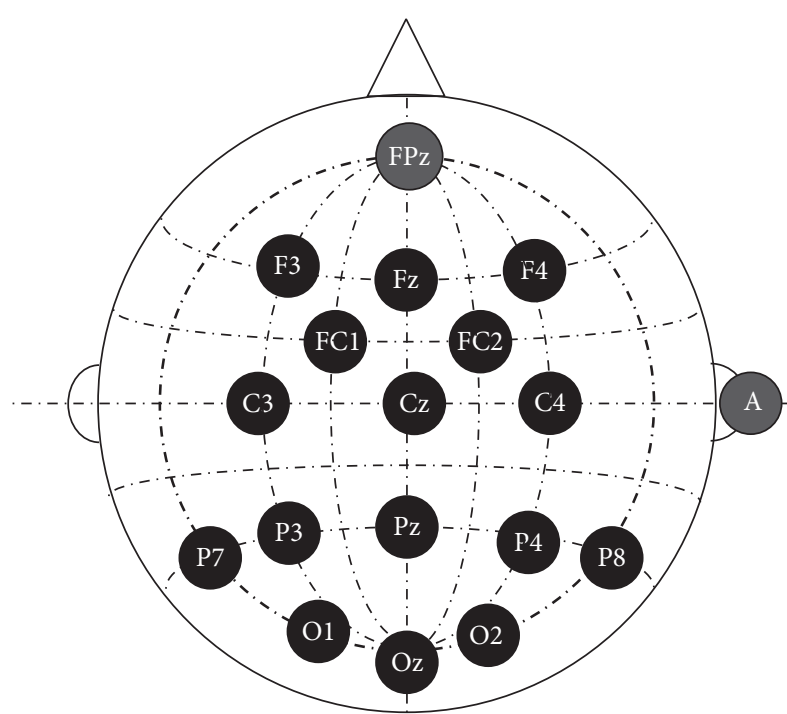

FIGURE 2: Configuration of the selected electrode positions (FPz was used as the ground electrode; right mastoid (A) was used as the reference electrode).

90th percentile were then replaced with the 10th percentile or the 90th percentile, respectively [19].

2.5. Classification Scheme. Bayesian linear discriminant analysis (BLDA) was used to build the classifier model for the online experiment. BLDA can effectively solve the problems of high-dimensional data sets or noise fitting owing to its regularization method. Hoffmann et al. [20] applied such a method to the classification of P300 BCIs and achieved good results.

2.6. Adaptive System Settings. The system was used to judge whether the results of two consecutive outputs were consistent. Accordingly, the final results of system output could be determined. If the results of two consecutive outputs were consistent, then the system exported the result as a feedback. Otherwise, the system would not provide any response until 16 trials were completed. When 16 trials ended, the classifier would automatically select the last output [21].

2.7. Statistical Analysis. We chose paired-samples $t$-tests (one-sample Kolmogorov-Smirnov test) for normal distribution to investigate the differences in mean amplitudes averaged from each ERP peak point \pm 20 ms between the F-P and STF-P paradigms. We also used such a method to explore the differences in online classification accuracy and bit rate between the two paradigms. The nonparametric Wilcoxon signed-rank test was used to compare the responses from the report of the subjects, as these data obey an uncertain distribution. The alpha level was $\alpha=0.05$.

2.8. Subjective Report. After finishing the tasks of two paradigms, we conducted a questionnaire survey of three questions. The three questions were answered by the subjects 

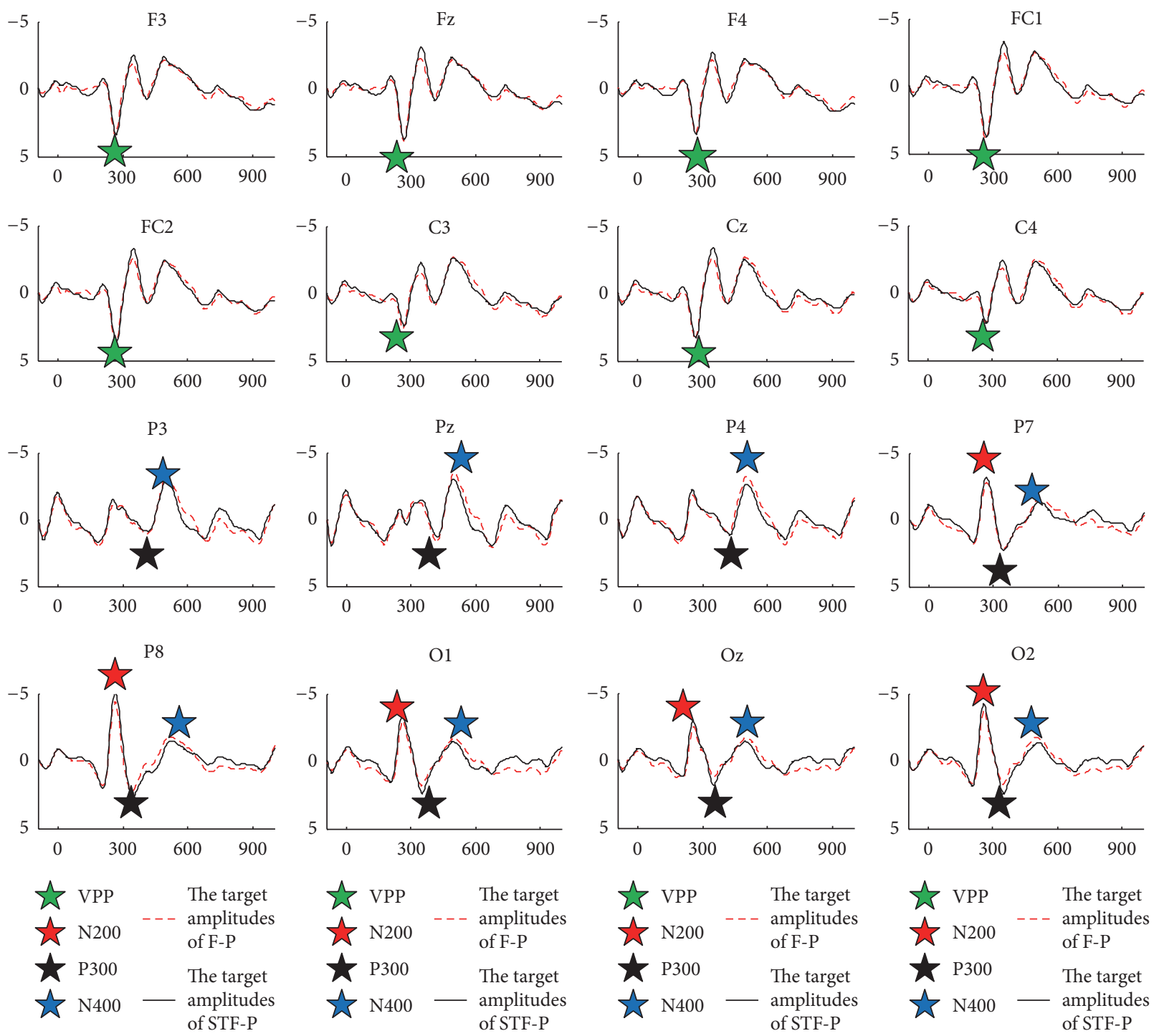

FIGURE 3: Grand averaged ERPs of target flashes across subjects 1-10 over 16 electrode sites.

on a 1-3 scale. A high score indicated a high degree of tiredness, difficulty, and annoyance (1: minimum; 2: medium; 3: maximum). The questions were as follows:

(1) Did this paradigm make you tired?

(2) Was this paradigm difficult?

(3) Did this paradigm make you annoyed?

\section{Results}

Figure 3 shows the grand averaged ERPs of target flashes after being baseline corrected by $100 \mathrm{~ms}$ prestimulus interval across subjects $1-10$ over 16 electrode sites [21]. The two paradigms had similar VPP components over frontal and central areas. However, a few differences were found in N200 and P300 over parietal and occipital sites. STF-P had relatively higher peak values across N200 and P300 than those of F-P over parietal and occipital sites. We explored the differences of VPP, N200, P300, and N400 between STF$\mathrm{P}$ and F-P; for this purpose, we selected $\mathrm{Cz}$ for VPP, P8 for $\mathrm{N} 200, \mathrm{Pz}$ for P300, and Cz for N400; these electrode positions typically contain the largest corresponding ERP components $[7,12,22,23]$ and are thus the best examples. Figure 4 shows the mean amplitudes of VPP at Cz, N200 at P8, P300 at Pz, and N400 at $\mathrm{Cz}$ for each subject and the N400 amplitude of the first and the third runs at $\mathrm{Cz}$ from the two paradigms [8]. The mean amplitude was averaged from each ERP peak point $\pm 20 \mathrm{~ms}$. The $\mathrm{N} 200$ amplitude at P8 from STF-P was significantly larger than that of F-P $(t=2.49, p<0.05, \mathrm{df}=$ 9 , Figure 4(b)). Furthermore, no significant difference was found between the two paradigms across VPP and P300 $(t=$ $0.35, p>0.05, \mathrm{df}=9$ for VPP, and $t=1.45, p>0.05$, $\mathrm{df}=$ 9 for P300, Figures 4(a) and 4(c)). Meanwhile, the value of P300 was smaller than that of the P300 reported in other studies $[19,24]$. Although the N400 at Cz from STF-P showed no significant difference compared with that from F-P $(t=$ $-0.65, p>0.05, \mathrm{df}=9$; Figure $4(\mathrm{~d}))$, the stability of N400 


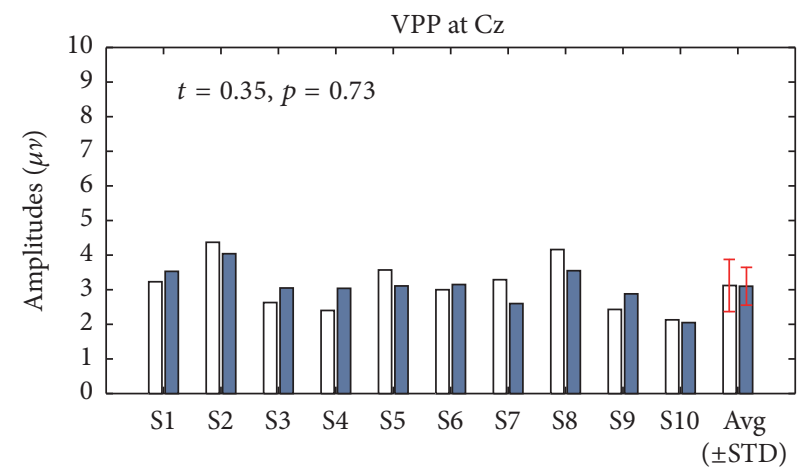

Subjects

$\square$ F-P

$\square$ STF-P

(a)

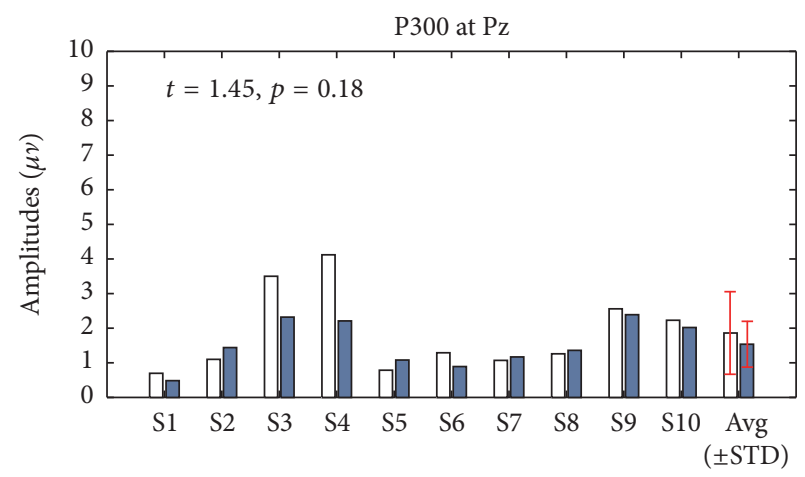

Subjects

$\square$ F-P

$\square$ STF-P

(c)

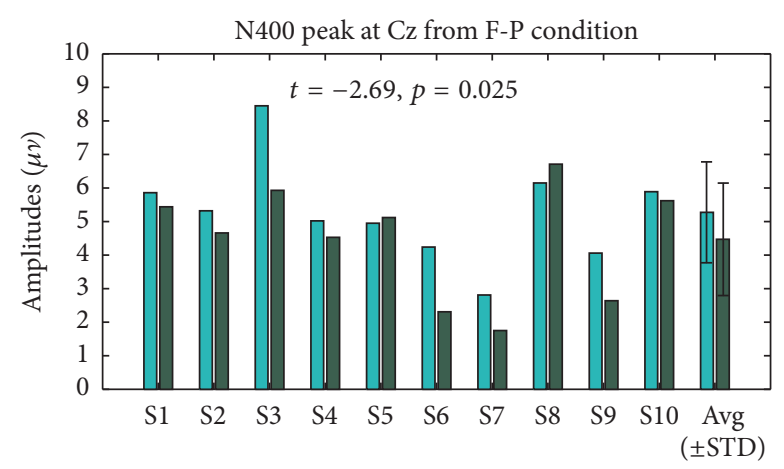

Subjects

$\square \mathrm{N} 400$ from the first offline run

口 N400 from the third offline run

(e)

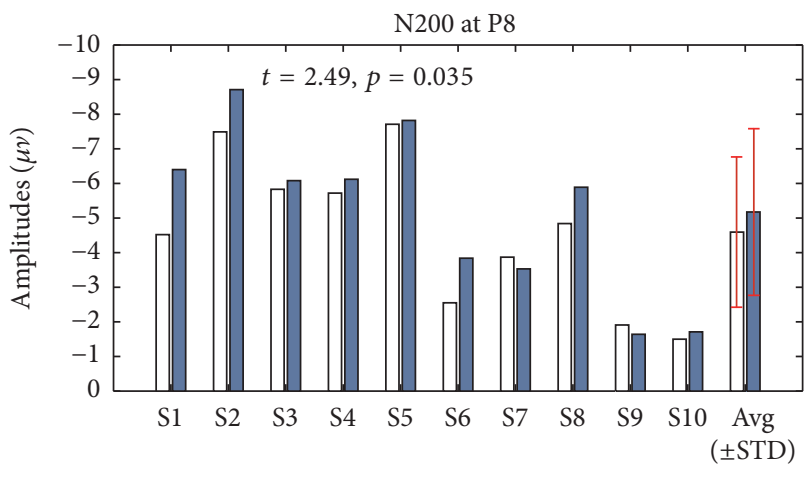

Subjects

$\square$ F-P

$\square$ STF-P

(b)

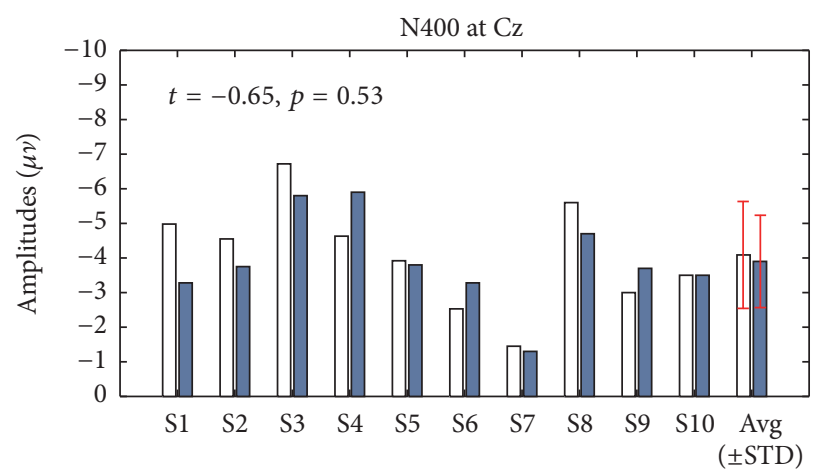

Subjects

$\square$ F-P

STF-P

(d)

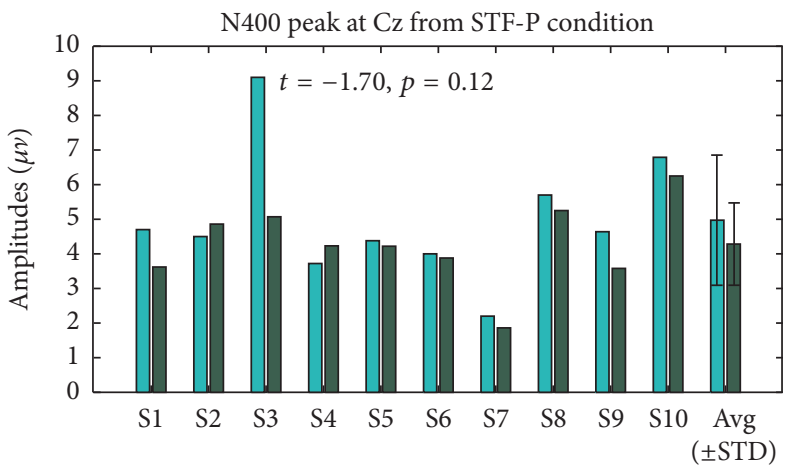

Subjects

$\square$ N400 from the first offline run

ㅁ N400 from the third offline run

(f)

FIGURE 4: Six panels presenting the mean amplitudes averaged from each ERP peak point $\pm 20 \mathrm{~ms}$ for each subject, and the differences in the amplitudes of $\mathrm{N} 400$ between the first and third offline runs from the two paradigms. These panels indicate the averaged amplitude of VPP at $\mathrm{Cz}$ (a); the averaged amplitude of $\mathrm{N} 200$ at P8 (b); the averaged amplitude of P300 at Pz (c); the averaged amplitude of N400 at Cz (d); the difference of N400 at Cz from the first and third offline run of the F-P (e); the difference of N400 at Cz from the first and third offline run of the STF-P (f). In addition, "Avg" is the average, "STD" is the standard deviation, and the error bars in the figure represent a standard deviation of each data set. 

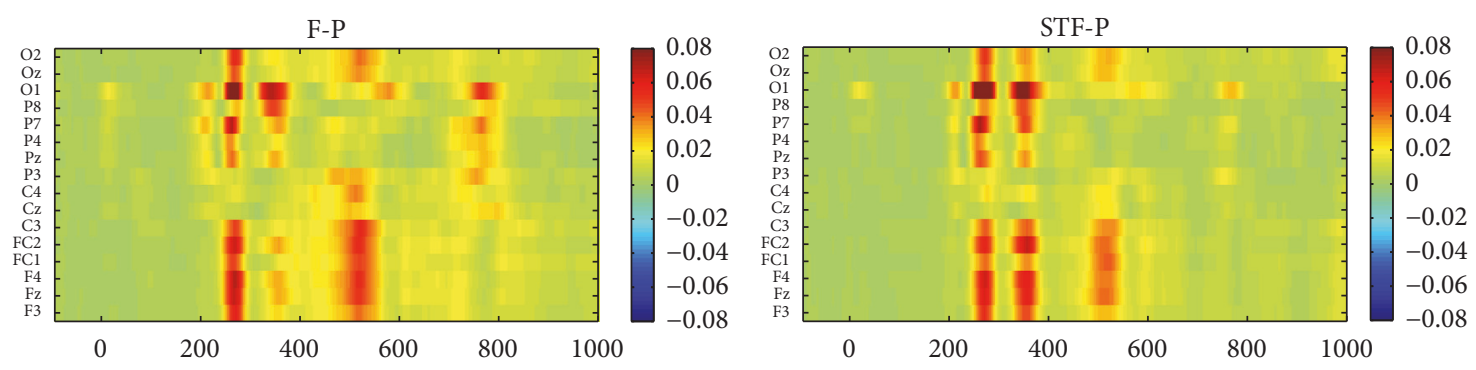

Figure 5: Absolute $R$-squared values of ERPs from these two patterns at 0-1000 ms averaged from subjects 1-10.
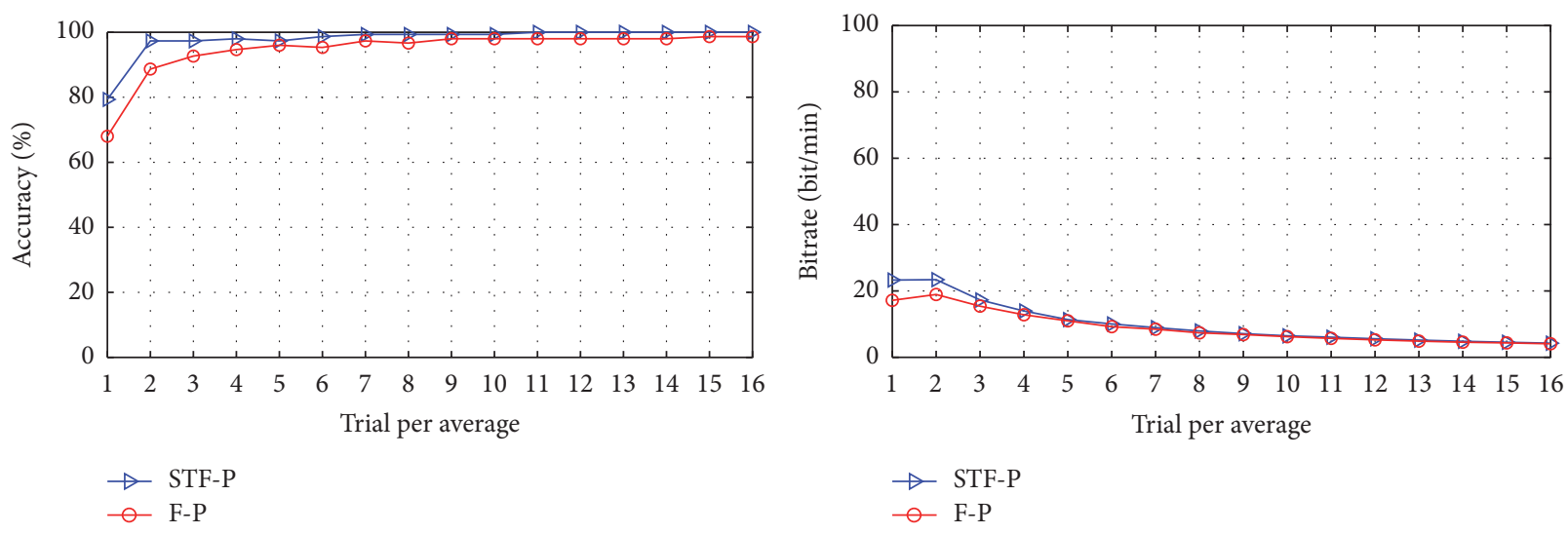

FIGURE 6: Classification accuracy and raw bit rate based on the offline data.

from STF-P $(t=-1.70, p>0.05, \mathrm{df}=9$; Figure 4(f)) was better than that from F-P $(t=-2.69, p<0.05, \mathrm{df}=9$; Figure $4(\mathrm{e}))$. Figure 5 shows the absolute $R$-squared values of ERPs from the two paradigms at $0-1000 \mathrm{~ms}$ averaged from subjects $1-10$ on 16 electrodes. $R$-squared values of ERPs reflected the time energy of the signals. The definition is as follows:

$$
r^{2}=\left(\frac{\sqrt{N_{1} N_{2}}}{N_{1}+N_{2}} \cdot \frac{\operatorname{mean}\left(X_{1}\right)-\operatorname{mean}\left(X_{2}\right)}{\operatorname{std}\left(X_{1} \cup X_{2}\right)}\right)^{2},
$$

where $X_{1}$ and $X_{2}$ are the features of classes " 1 " and " 2 ," respectively and $N_{1}$ and $N_{2}$ are the number of samples.

Figure 6 shows the classification accuracy and raw bit rate based on the offline data [8]. The values were obtained from 15 -fold cross-validation. The classification accuracy and bit rate of STF-P were better than those of F-P when 1-16 numbers of trials were used for averaging.

Figure 7 shows the contributions of N200 (between 150 and $300 \mathrm{~ms}$ ), P300 (between 300 and $450 \mathrm{~ms}$ ), and N400 (between 450 and $700 \mathrm{~ms}$ ) on the BCI performance [8]. The two graphs indicated that N200 and P300 components were crucial in the classification accuracy. In addition, the N400 component also contributed to the classification accuracy.

Table 2 shows the online classification accuracy, bit rate, and mean number of trials for each subject. The classification accuracy and bit rate of STF-P were significantly higher than those of F-P $(t=2.89, p<0.05$, df $=9$ for classification accuracy, $t=4.03, p<0.05, \mathrm{df}=9$ for bit rate). Moreover, the number of trials for averaging of STF-P was significantly less than that of F-P $(t=-2.33, p<0.05, \mathrm{df}=9)$.

Table 3 presents the responses of the subjects to the three questions for each paradigm. We further investigated the differences between the two paradigms. For this purpose, we chose the Wilcoxon signed-rank test method owing to the fact that the data satisfy an uncertain distribution. No significant differences were found between the two paradigms in terms of the degree of tiredness $(p>0.05)$, degree of difficulty $(p>0.05)$, and degree of annoyance $(p>0.05)$.

\section{Discussion}

This study aimed to survey whether any difference would be found between STF-P in which the subject could see the target character during the time the stimuli were on and F-P in which the target character was concealed during the time the stimuli were on. The results showed that the STF-P could elicit larger N200 component and improve the classification accuracy and bit rate of the BCI system compared with the F-P.

The Eriksen flanker task [25] is a commonly used experimental design to obtain N200 and is a kind of a mismatch paradigm [21]. In the present study, the STF-P elicited larger N200 component than the F-P. On the one hand, semitransparent face stimuli may lead to a high mismatch, thereby resulting in a large N200. On the other hand, the psychological salience of the stimuli can be exploited to elicit high ERP components not confined to the P300 components [9]. 


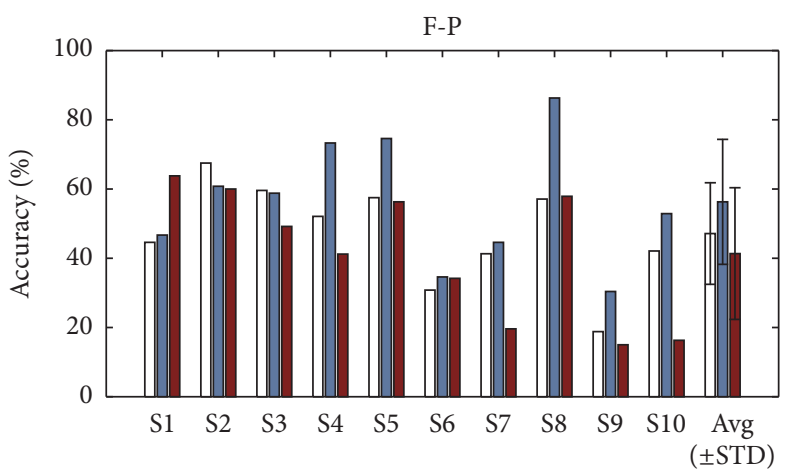

Subjects

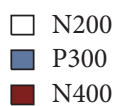

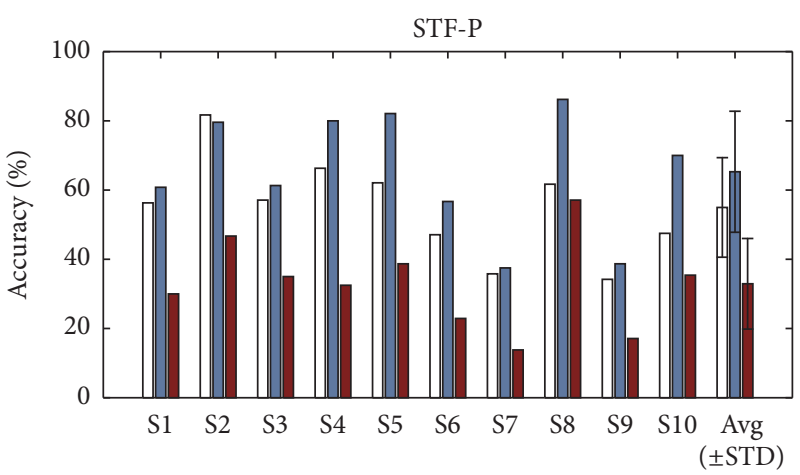

Subjects

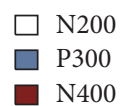

(a)

(b)

FIgURE 7: The contributions of N200, P300, and N400 time windows on the classification accuracy.

TABLE 2: Online classification accuracy, bit rate, and average number of trials for each subject.

\begin{tabular}{|c|c|c|c|c|c|c|c|c|c|c|c|c|}
\hline & & S1 & S2 & S3 & S4 & S5 & S6 & S7 & S8 & S9 & S10 & $\mathrm{AVG} \pm \mathrm{STD}$ \\
\hline \multirow{2}{*}{$\operatorname{ACC}(\%)$} & F-P & 94.4 & 94.4 & 91.7 & 94.4 & 80.5 & 100 & 94.4 & 77.8 & 91.7 & 100 & $91.9 \pm 7.3$ \\
\hline & STF-P & 97.2 & 94.4 & 97.2 & 100 & 88.9 & 97.2 & 97.2 & 83.3 & 94.4 & 100 & $95.0 \pm 5.2$ \\
\hline \multirow{2}{*}{ RBR (bit/min) } & F-P & 32.9 & 42.7 & 36.3 & 44.5 & 32.3 & 44.3 & 43.9 & 29.4 & 28.6 & 44.8 & $38.0 \pm 6.7$ \\
\hline & STF-P & 41.0 & 44.5 & 43.0 & 50.3 & 39.3 & 43.0 & 42.5 & 36.1 & 36.6 & 49.6 & $42.6 \pm 4.8$ \\
\hline \multirow{2}{*}{ AVT } & F-P & 2.78 & 2.14 & 2.39 & 2.06 & 2.14 & 2.33 & 2.08 & 2.22 & 3.03 & 2.31 & $2.35 \pm 0.32$ \\
\hline & STF-P & 2.36 & 2.06 & 2.25 & 2.06 & 2.08 & 2.25 & 2.28 & 2.03 & 2.50 & 2.08 & $2.20 \pm 0.16$ \\
\hline
\end{tabular}

$\mathrm{ACC}=$ classification accuracy, $\mathrm{RBR}=$ raw bit rate $(\mathrm{bit} / \mathrm{min}), \mathrm{AVT}=$ average number of trials used to classify each character, STF-P $=$ semitransparent face pattern, $\mathrm{F}-\mathrm{P}=$ face pattern, $\mathrm{AVG}=$ average, and $\mathrm{STD}=$ standard deviation.

TABLE 3: Subjects' responses to three questions for each pattern.

\begin{tabular}{lcccccccccccc}
\hline & & S1 & S2 & S3 & S4 & S5 & S6 & S7 & S8 & S9 & S10 & AVG \pm STD \\
\hline \multirow{2}{*}{ Tired } & F-P & 2 & 2 & 2 & 1 & 2 & 2 & 1 & 2 & 1 & 1 & $1.6 \pm 0.52$ \\
& STF-P & 1 & 2 & 1 & 2 & 1 & 1 & 1 & 1 & 1 & 1 & $1.2 \pm 0.42$ \\
Difficult & F-P & 2 & 1 & 1 & 2 & 1 & 1 & 2 & 2 & 1 & 2 & $1.5 \pm 0.53$ \\
& STF-P & 1 & 2 & 1 & 1 & 1 & 2 & 1 & 2 & 1 & 1 & $1.3 \pm 0.48$ \\
Annoyed & F-P & 1 & 2 & 1 & 2 & 1 & 1 & 1 & 2 & 1 & 1 & $1.3 \pm 0.48$ \\
& STF-P & 1 & 1 & 1 & 2 & 1 & 1 & 1 & 2 & 1 & 1 & $1.2 \pm 0.42$
\end{tabular}

$\mathrm{F}-\mathrm{P}=$ face pattern; STF-P = semitransparent face pattern. AVG is average and STD is standard deviation.

The difference of $\mathrm{P} 300$ amplitude between two paradigms in this paper was not clear and no significant difference was found. This phenomenon may be attributed to the low luminosity contrast when the background is black (the gray value of face was set 110, while the background was 255). However, low luminosity contrast leads to low visual fatigue. Li et al. [26] studied the effects of various luminosity contrasts on the BCI performance and found that low luminosity contrast produces small amplitude for P300 on average. A high luminosity contrast can result in bright, noticeable infrequent stimuli; as a result, subjects can easily concentrate their attention and efficiently identify the target characters. Therefore, low brightness may lead to increased effort or attention deployment in subjects. This finding may have an important implication for clinical application.

The effects of repetition can decrease the amplitude of N400, especially for long-term offline data recording [8]. In STF-P, given that the target differed, the stimulus also differed when the subject shifted their focus from one target to another. Since the significant difference of N400 between 
the first and third offline run was found in the F-P while not in the STF-P (see Figures 4(e) and 4(f)), it indicated that STF-P contained less repetition effects compared to the F-P.

Classification accuracy and ITR are the important indexes of BCI performance. In previous studies, face paradigms themselves have had good performances in accuracy (mean accuracy was higher than 90\%) [8]. Therefore, even $1 \%$ increment in accuracy would be a good improvement. In this study, the averaged classification accuracy and bit rate of the STF-P were $95.0 \%, 42.6 \mathrm{bit} / \mathrm{min}$, while those of the F-P were $91.9 \%, 38.0 \mathrm{bit} / \mathrm{min}$, and were $3.1 \%, 4.6 \mathrm{bit} / \mathrm{min}$, higher than those of the F-P. Figure 4(b) showed that the N200 at P8 of the STF-P was significantly higher than that of the F-P. Figure 7 showed that N200 could contribute to classification accuracy. Kaufmann et al. [27] reported that the potential of N200 can enhance the classification accuracy. Jin et al. [8] proved that other components can contribute to the classification accuracy under the condition of small P300 amplitudes. Table 2 showed that the classification accuracy and information transfer rate of the STF-P were significantly higher than that of the F-P $(p<0.05)$. As in all, the STF-P could obtain superior performance compared to the F-P.

This research studied two paradigms only (semitransparency and nontransparency) and focused less on the different transparent degrees based on the state of being transparent. However, this research provided a new idea on the studies of face stimuli and demonstrated that other distinct components could contribute strongly to the BCI performance.

\section{Conclusion}

In this study, we measured the performance of STF-P and F-P on BCI. The result indicated that STF-P was superior to F-P. In future studies, we will further verify the performance of the STF-P pattern on patients.

\section{Conflicts of Interest}

The authors declare that they have no conflicts of interest.

\section{Acknowledgments}

This work was supported in part by the Grant National Natural Science Foundation of China, under Grants nos. 61573142, 61203127, 91420302, and 61305028. This work was also supported by the Fundamental Research Funds for the Central Universities (WG1414005, WH1314023, and WH1516018).

\section{References}

[1] E. W. Sellers, D. J. Krusienski, D. J. McFarland, T. M. Vaughan, and J. R. Wolpaw, "A P300 event-related potential braincomputer interface (BCI): the effects of matrix size and inter stimulus interval on performance," Biological Psychology, vol. 73, no. 3, pp. 242-252, 2006.

[2] F. Nijboer, E. W. Sellers, J. Mellinger et al., "A P300-based brain-computer interface for people with amyotrophic lateral sclerosis," Clinical Neurophysiology, vol. 119, no. 8, pp. 1909-1916, 2008.
[3] X.-Y. Wang, F. Cai, J. Jin, Y. Zhang, and B. Wang, "Robot control system based on auditory brain-computer interface," Control Theory and Applications, vol. 32, no. 9, pp. 1183-1190, 2015.

[4] M. F. Mason, M. I. Norton, J. D. Van Horn, D. M. Wegner, S. T. Grafton, and C. N. Macrae, "Wandering minds: the default network and stimulus-independent thought," Science, vol. 315, no. 5810, pp. 393-395, 2007.

[5] A. Kübler, B. Kotchoubey, J. Kaiser, J. R. Wolpaw, and N. Birbaumer, "Brain-computer communication: unlocking the locked in," Psychological Bulletin, vol. 127, no. 3, pp. 358-375, 2001.

[6] J. R. Wolpaw, N. Birbaumer, D. J. McFarland, G. Pfurtscheller, and T. M. Vaughan, "Brain-computer interfaces for communication and control," Clinical Neurophysiology, vol. 113, no. 6, pp. 767-791, 2002.

[7] L. A. Farwell and E. Donchin, "Talking off the top of your head: toward a mental prosthesis utilizing event-related brain potentials," Electroencephalography and Clinical Neurophysiology, vol. 70, no. 6, pp. 510-523, 1988.

[8] J. Jin, B. Z. Allison, Y. Zhang, X. Wang, and A. Cichocki, "An erp-based bci using an oddball paradigm with different faces and reduced errors in critical functions," International Journal of Neural Systems, vol. 24, no. 8, Article ID 1450027, 2014.

[9] T. Kaufmann, S. M. Schulz, C. Grünzinger, and A. Kübler, "Flashing characters with famous faces improves ERP-based brain-computer interface performance," Journal of Neural Engineering, vol. 8, no. 5, pp. 173-178, 2011.

[10] J. Jin, B. Z. Allison, T. Kaufmann et al., "The changing face of P300 BCIs: a comparison of stimulus changes in a P300 BCI involving faces, emotion, and movement," PLoS ONE, vol. 7, no. 11, Article ID e49688, 2012.

[11] Y. Zhang, Q. Zhao, J. Jin, X. Y. Wang, and A. Cichocki, "A novel BCI based on ERP components sensitive to configural processing of human faces," Journal of Neural Engineering, vol. 9, no. 2, pp. 26018-26030, 2012.

[12] D. A. Jeffreys and E. S. A. Tukmachi, "The vertex-positive scalp potential evoked by faces and by objects," Experimental Brain Research, vol. 91, no. 2, pp. 340-350, 1992.

[13] T. Kaufmann, S. M. Schulz, A. Köblitz, G. Renner, C. Wessig, and A. Kübler, "Face stimuli effectively prevent brain-computer interface inefficiency in patients with neurodegenerative disease," Clinical Neurophysiology, vol. 124, no. 5, pp. 893-900, 2013.

[14] S.-K. Yeom, S. Fazli, K.-R. Müller, and S.-W. Lee, "An efficient ERP-based brain-computer interface using random set presentation and face familiarity," PLOS ONE, vol. 9, no. 11, Article ID el11157, 2014

[15] J. Jin, P. Horki, C. Brunner, X. Wang, C. Neuper, and G. Pfurtscheller, "A new P300 stimulus presentation pattern for EEG-based spelling systems," Biomedizinische Technik, vol. 55, no. 4, pp. 203-210, 2010.

[16] J. Jin, B. Z. Allison, E. W. Sellers et al., "Optimized stimulus presentation patterns for an event-related potential EEG-based brain-computer interface," Medical and Biological Engineering and Computing, vol. 49, no. 2, pp. 181-191, 2011.

[17] A. Lenhardt, M. Kaper, and H. J. Ritter, "An adaptive P300-based online brain-computer interface," IEEE Transactions on Neural Systems and Rehabilitation Engineering, vol. 16, no. 2, pp. 121130, 2008.

[18] J. Jin, B. Z. Allison, E. W. Sellers et al., "An adaptive P300-based control system," Journal of Neural Engineering, vol. 8, no. 3, Article ID 036006, pp. 292-301, 2011. 
[19] J. Jin, I. Daly, Y. Zhang, X. Y. Wang, and A. Cichocki, "An optimized ERP brain-computer interface based on facial expression changes," Journal of Neural Engineering, vol. 11, no. 3, pp. 10821088, 2014.

[20] U. Hoffmann, J.-M. Vesin, T. Ebrahimi, and K. Diserens, "An efficient P300-based brain-computer interface for disabled subjects," Journal of Neuroscience Methods, vol. 167, no. 1, pp. 115-125, 2008.

[21] J. Jin, E. W. Sellers, S. Zhou, Y. Zhang, X. Wang, and A. Cichocki, "A P300 brain-computer interface based on a modification of the mismatch negativity paradigm," International Journal of Neural Systems, vol. 25, no. 3, pp. 595-599, 2015.

[22] J. R. Folstein and C. Van Petten, "Influence of cognitive control and mismatch on the N2 component of the ERP: a review," Psychophysiology, vol. 45, no. 1, pp. 152-170, 2008.

[23] C. C. Duncan, R. J. Barry, J. F. Connolly et al., "Eventrelated potentials in clinical research: guidelines for eliciting, recording, and quantifying mismatch negativity, P300, and N400," Clinical Neurophysiology, vol. 120, no. 11, pp. 1883-1908, 2009.

[24] L. Chen, J. Jin, Y. Zhang, X. Y. Wang, and A. Cichocki, "A survey of the dummy face and human face stimuli used in BCI paradigm," Journal of Neuroscience Methods, vol. 239, pp. 18-27, 2015.

[25] B. A. Eriksen and C. W. Eriksen, "Effects of noise letters upon the identification of a target letter in a nonsearch task," Perception and Psychophysics, vol. 16, no. 1, pp. 143-149, 1974.

[26] Y. Li, S. Bahn, C. S. Nam, and J. Lee, "Effects of luminosity contrast and stimulus duration on user performance and preference in a P300-based brain-computer interface," International Journal of Human-Computer Interaction, vol. 30, no. 2, pp. 151163, 2014.

[27] T. Kaufmann, E. M. Hammer, and A. Kübler, "ERPs contributing to classification in the P300 BCI," in Proceedings of the 5th International Brain-Computer Interface Conference, pp. 136-139, 2013. 

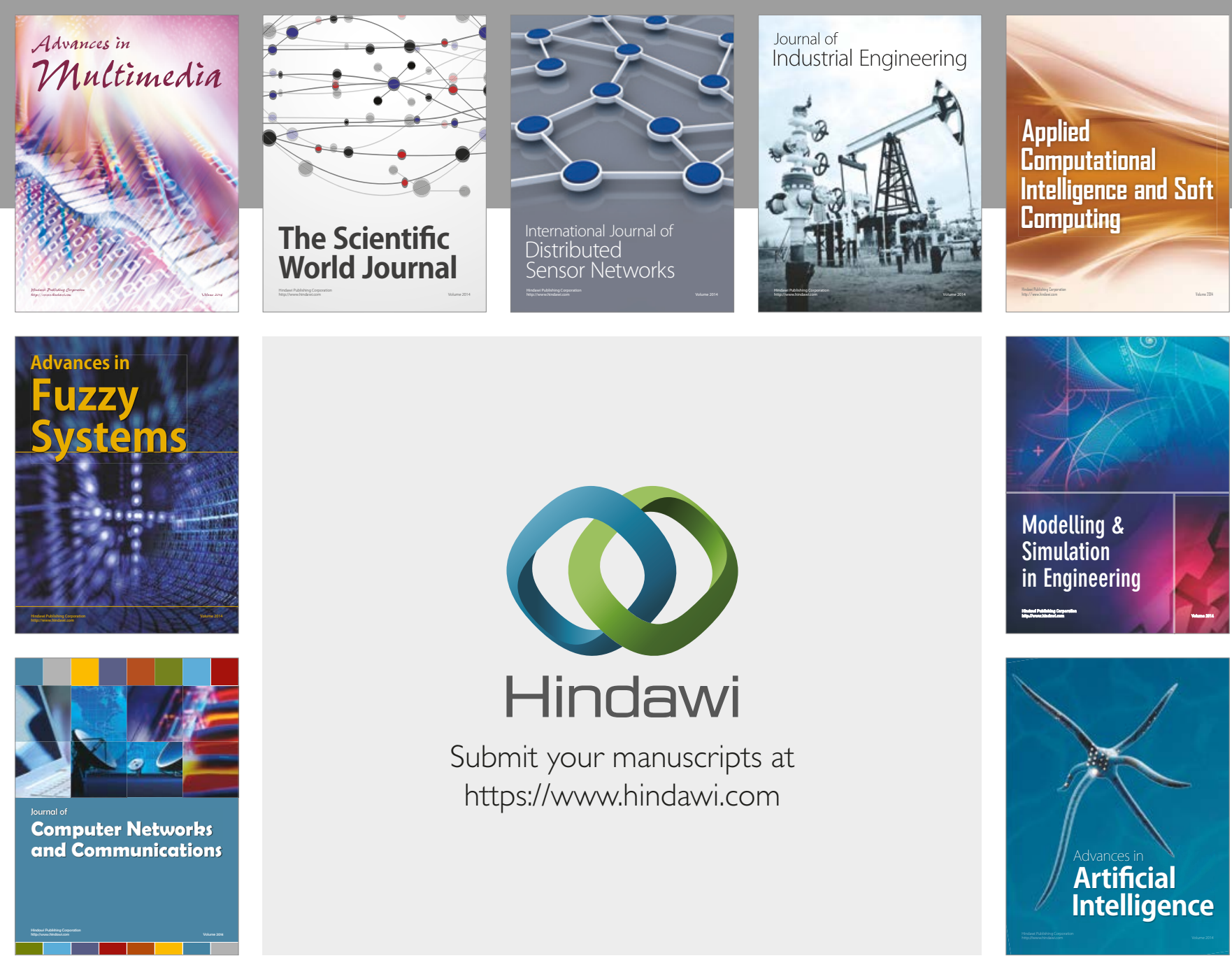

\section{Hindawi}

Submit your manuscripts at

https://www.hindawi.com
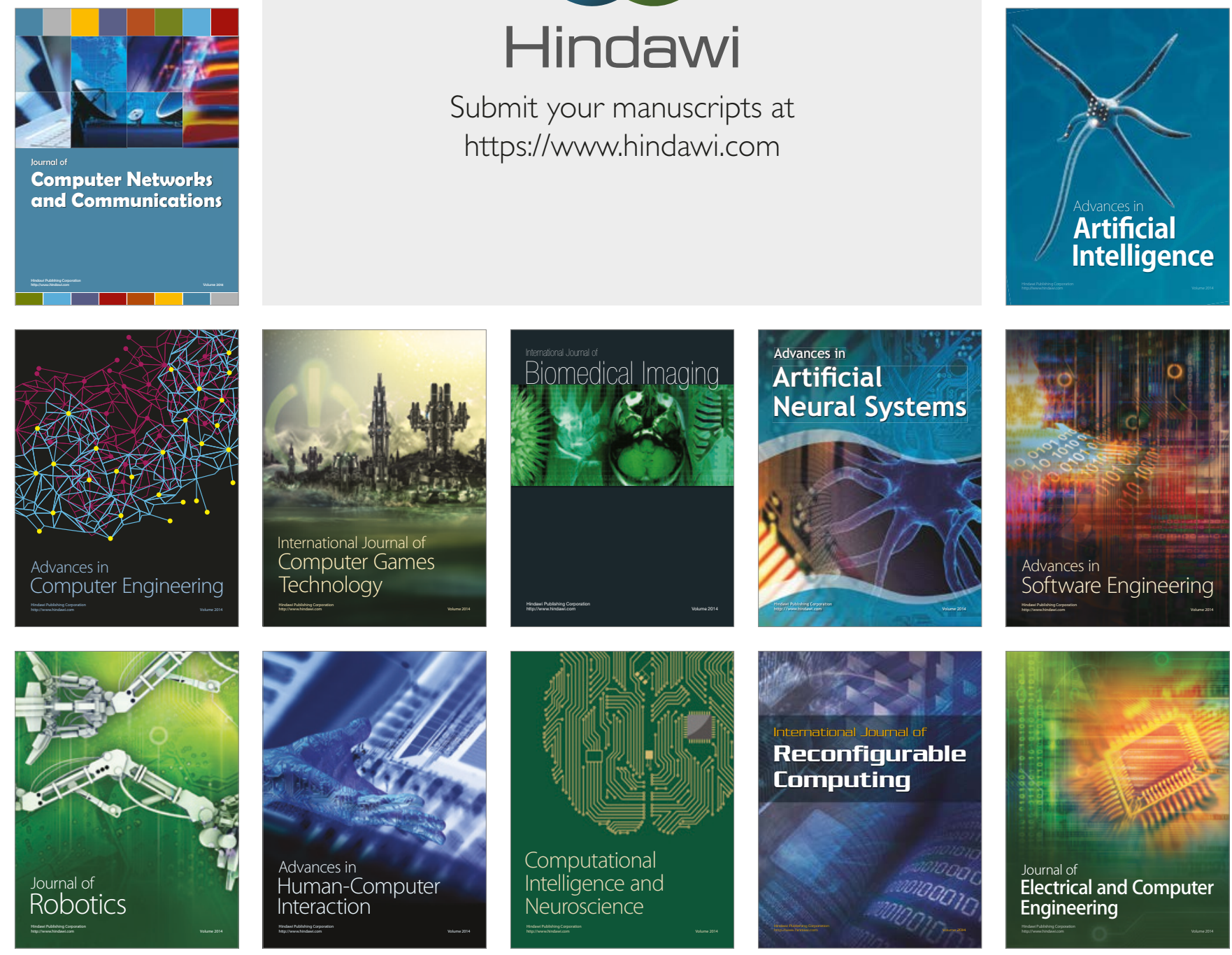